\title{
Priorités pour l'éducation des adolescentes
}

Cynthia B. Lloyd

Population Council

Follow this and additional works at: https://knowledgecommons.popcouncil.org/departments_sbsr-pgy

Part of the Family, Life Course, and Society Commons, Gender and Sexuality Commons, Gender Equity in Education Commons, and the International Public Health Commons How does access to this work benefit you? Let us know!

\section{Recommended Citation}

Lloyd, Cynthia B. 2012. "Priorités pour l'éducation des adolescentes," GIRLS FIRST! Perspectives on GirlCentered Programming. New York: Population Council. 


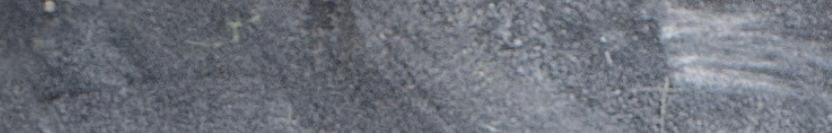

UNE PUBLICATION GIRLS FIRST!

PRIORITÉS POUR L'ÉDUCATION

DES

ADOLESCENTES

\section{PAR CYNTHIA B. LLOYD}

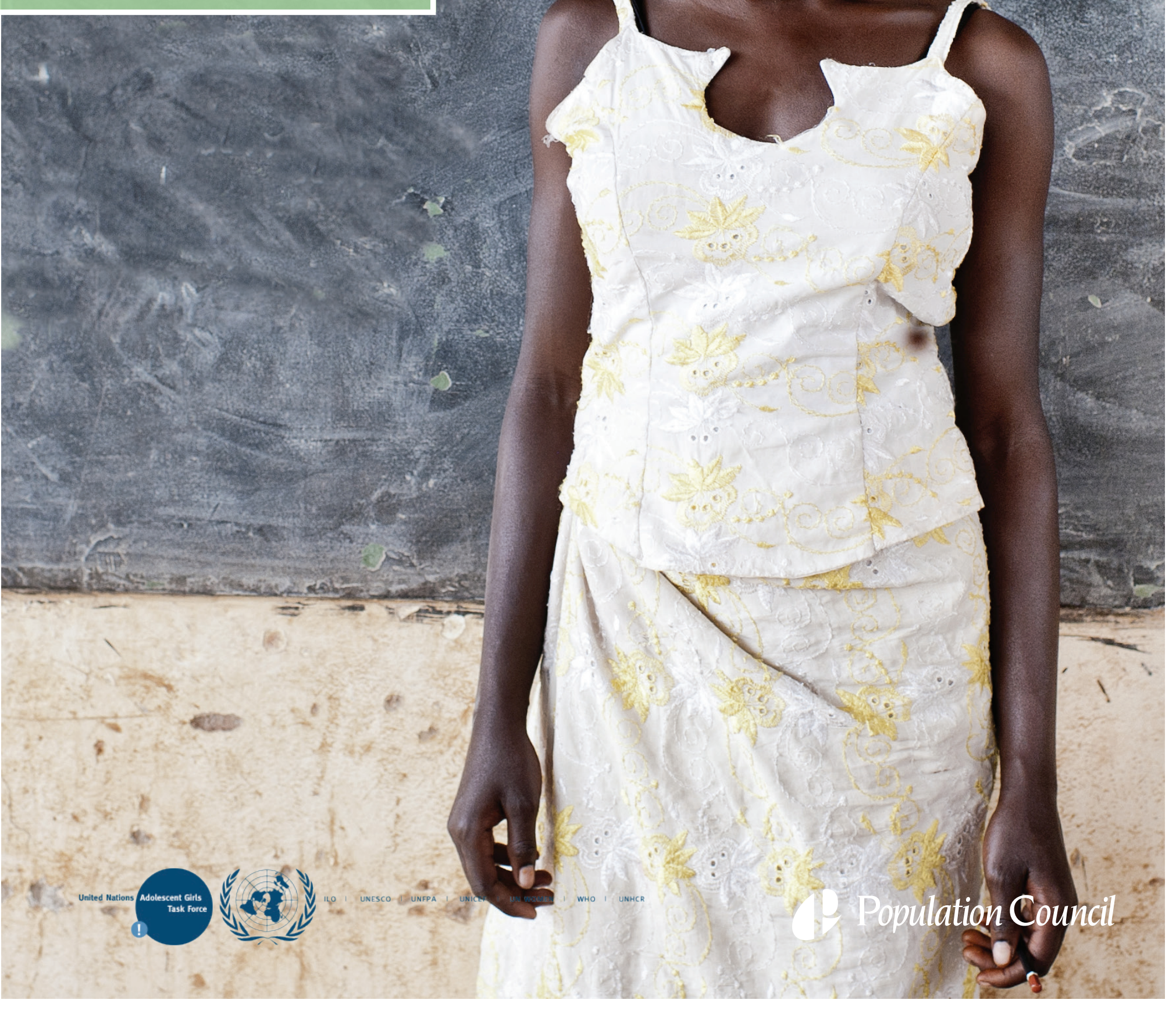

\section{6}

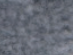

BILAN

POLITIQUE/

PROGRAMMATION 


\section{GIRLS FIRST!}

\section{PERSPECTIVES DE PROGRAMMATION CENTRÉE SUR LES FILLES}

La recherche et les programmes consacrés aux adolescentes se concentrent traditionnellement sur la sexualité, la santé génésique et le comportement, négligeant la problématique socioéconomique plus large qui sous-tend leurs droits humains, leur développement général, leur santé et leur bienêtre. De plus, les efforts d'amélioration de la vie des filles mettent souvent en vedette ceux et celles qui dominent ou influencent leur vie (les parents, la belle-famille, les garçons, les hommes, les agresseurs), sans prêter aux intéressées elles-mêmes l'attention qu'elles méritent en soi.

GIRLS FIRST! Perspectives de programmation centrée sur les filles est une série de cinq comptes rendus thématiques, rédigés par les experts et expertes du Population Council. Ces dossiers présentent un instantané de la base de connaissances telle qu'elle se présente, à un moment donné, dans ce domaine en évolution permanente. Ils abordent les cinq priorités stratégiques définies dans la Déclaration conjointe des Nations Unies, "Intensifier l'action menée pour promouvoir les droits des adolescentes " (mars 2010), symbole de l'engagement collectif de sept organismes de l'ONU au soutien des gouvernements et de leurs partenaires pour la promotion de politiques et programmes essentiels au profit des adolescentes les plus difficiles à atteindre. Ces comptes rendus

1. explorent l'évolution prochaine de l'éducation des filles;

2. définissent les grandes lignes d'approches innovantes en vue de l'amélioration de la santé des filles;

3. recadrent l'approche de la violence à l'encontre des filles ;

4. décrivent les meilleurs moyens de cultiver les leaders féminines; et

5. expliquent de nouveaux modes inédits de collecte et d'exploitation des données relatives aux adolescentes.

Ils avancent des arguments novateurs en faveur de l'investissement dans les filles et mettent en valeur les pratiques prometteuses. Ils expriment un point de vue factuel progressiste quant aux allocations de ressources aptes à assurer le plus rapidement et le plus efficacement l'amélioration de la vie des filles.

Rédigés par des experts et expertes du Population Council, une organisation pionnière d'une recherche et programmation de pointe à l'intention des adolescentes vulnérables et marginalisées, ces rapports ont bénéficié, d'abord et surtout, de l'appui et du leadership de l'Équipe spéciale des Nations Unies pour les adolescentes. L'initiative a également reçu l'appui moral et financier de la Nike Foundation, de la Fondation des Nations Unies et de la David and Lucile Packard Foundation. Ces comptes rendus viennent s'ajouter à la guidance programmatique et aux " boîtes à outils " aujourd'hui disponibles ; ils offrent une ressource de référence essentielle à toute initiative d'élaboration de politiques et programmes efficaces et durables au profit des filles. Notre espoir est qu'ils inspireront de nouvelles approches utiles aux efforts de réalisation des droits des adolescentes marginalisées du monde.

Décembre 2011 Population Council et Équipe spéciale des Nations Unies pour les adolescentes

Avis

Ces comptes rendus sur la programmation pour adolescentes dans les domaines de l'éducation, de la santé, de la réduction de la violence, du leadership des filles et des données ont été préparés par le Population Council pour l'Équipe spéciale des Nations Unies pour les adolescentes, avec l'appui de la Fondation des Nations Unies, de la Nike Foundation et de la Packard Foundation.

Les opinions exprimées sont strictement celles des auteurs et ne reflètent pas nécessairement celles des partenaires donateurs. 


\section{PRIORITÉS POUR L'EDUCATION DES ADOLESCENTES}

PAR CYNTHIA B. LLOYD

\section{CONNAISSANCES ET PRINCIPES FONDAMENTAUX ......................................................... 2}

APPROCHES PROMETTEUSES À TESTER ET À DÉVELOPPER ........................................ 2

Programmes d'éducation non formelle pour adolescentes déscolarisées .......................................... 2

Établir et renforcer les filières de scolarisation non formelle à formelle ; mettre l'accent sur les cadettes

Élargir le soutien de solutions d'éducation non formelle à l'objectif

de compétences utiles.

Étendre l'admissibilité aux subventions conditionnelles et aux bourses (sur la base du besoin et du mérite) aux filles inscrites aux programmes non formels réputés ............................................4

Collecter les données d'impact des programmes d'éducation non formelle et les évaluer.................6

Politiques et programmes d'éducation utiles aux adolescentes scolarisées ....................................6

Encourager l'alignement de l'âge sur le niveau scolaire approprié ....................................................6

Rendre les écoles primaires et secondaires formelles plus favorables aux filles .............................6

Créer des programmes d'activités périscolaires réservés aux filles .................................................6 6

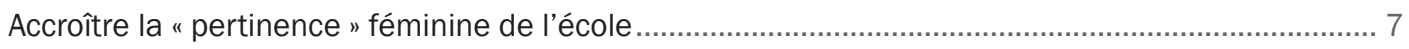

Établir un niveau d'enseignants d'élite, formés et rémunérés davantage, ayant fait preuve de compétences dans les matières " pertinentes " ............................................ 7

RESSOURCES : PUBLICATIONS ET PROGRAMMES DIGNES D'INTÉRÊT ............................. 7

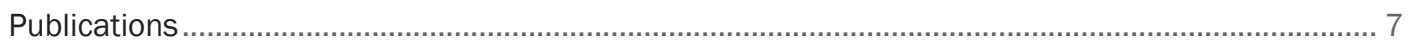

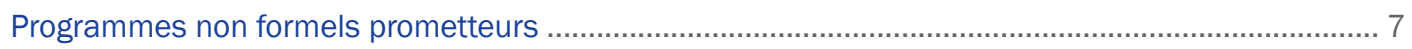

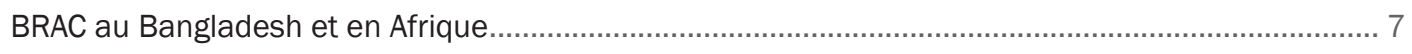

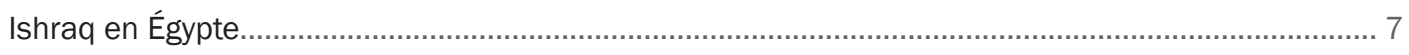

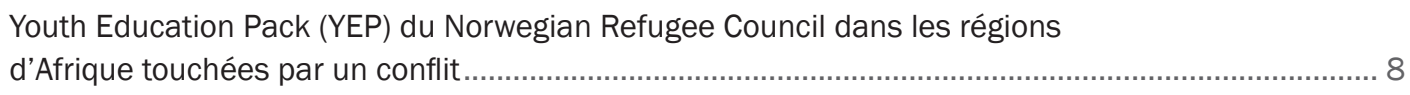

Programmes prometteurs au soutien d'une scolarisation formelle pertinente

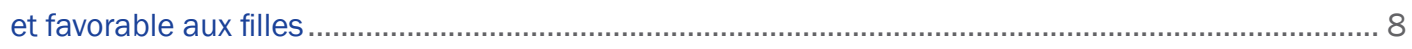

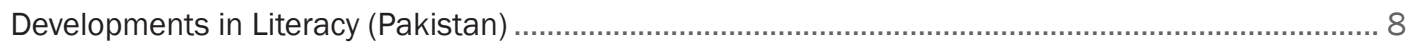

Camfed (Campaign for female education) en Afrique .............................................................. 8

Programmes de scolarisation des filles Room to Read en Asie et en Afrique ..................................... 8

Forum for African Women Educationalists - Forum des Éducatrices africaines (FAWE),
Écoles sensibles au genre et centres d'excellence en Afrique $\ldots \ldots \ldots \ldots \ldots \ldots \ldots \ldots \ldots \ldots \ldots \ldots \ldots \ldots \ldots \ldots \ldots \ldots \ldots \ldots \ldots \ldots \ldots \ldots \ldots \ldots \ldots \ldots \ldots \ldots \ldots \ldots \ldots \ldots \ldots \ldots$

Japan Relief for Cambodia/American Assistance for Cambodia (JRfC/AAfC)

Girls be Ambitious (Cambodge). 


\section{CONNAISSANCES ET PRINCIPES FONDAMENTAUX}

L'adolescence, plus précoce chez les filles que chez les garçons, marque une période de croissance et de développement rapides sur le plan physique, affectif et cognitif. Côté féminin, il s'agit d'une étape de la vie durant laquelle l'éducation, si elle est efficace, peut être transformatrice. Mais il s'agit aussi du moment où les filles sont le plus susceptibles d'abandonner l'école.

Un problème majeur, dans la plupart des pays en développement, est que les systèmes d'éducation en place ne cernent et ne réalisent pas leur potentiel d'autonomisation des adolescentes en les dotant de compétences économiquement productives. L'éducation des filles durant l'adolescence peut les tenir à l'abri des risques d'une initiation sexuelle précoce, différer le mariage et la maternité et leur permettre de vivre une enfance comparable à celle des garçons, libres du fardeau de travaux ménagers excessifs. Pour les adolescentes, une éducation de qualité peut aussi être source de connaissances accrues et de meilleures compétences, gages d'une vie active rémunératrice et d'un engagement communautaire efficace. Parce que les filles atteignent leur maturité physique et sexuelle alors qu'elles sont encore enfants (et donc vulnérables), pour être de qualité, leur éducation dans le secteur formel ou non doit être à la fois protectrice, favorable au sexe féminin et autonomisante.

Les écoles ont le potentiel d'en faire plus pour habiliter les filles à traduire les connaissances et compétences acquises en une situation d'emploi rémunérateur, de meilleure santé et de citoyenneté active sur le long terme. À l'heure où l'écart entre les inscriptions scolaires et niveaux d'études atteints se comble rapidement entre les deux sexes, il demeure inchangé sur le plan de la participation à la vie active (Lloyd 2011). La Figure 1 illustre le contraste entre le faible écart de scolarisation entre les garçons et les filles, en fonction de l'âge, dans quatre grandes régions du monde et l'écart grandissant, dans trois de ces régions, en termes de participation à la vie active tandis que les adolescents quittent l'école et approchent l'âge adulte. Elle révèle aussi les très faibles niveaux de participation à la vie active, en fonction de l'âge et pour les deux sexes, en Afrique subsaharienne. Pour que les écoles contribuent à l'établissement durable de l'équité sociale entre les sexes, il importe qu'elles profitent pour le moins autant aux filles qu'aux garçons.

L'inquiétude grandissante suscitée par la situation précaire des filles pauvres du monde en développement ces 15 dernières années a donné lieu à un large éventail d'interventions programmatiques conçues à leur avantage. Pour la plupart, les programmes mis au point en dehors du contexte éducatif formel comportent un élément pédagogique. L'attention aux besoins des filles adolescentes en particulier est plus récente. Axée principalement sur les filles sans scolarisation aucune ou déscolarisées prématurément, cette attention les a laissées insuffisamment préparées à l'âge adulte et vulnérables aux risques sexuels ainsi que du mariage et de la maternité précoces. L'attention aux besoins des adolescentes restées à l'école s'est avérée moindre, bien que la situation commence à changer. Ces deux populations adolescentes - les filles actuellement scolarisées et celles qui ne le sont plus ou ne l'ont jamais été - présentent des besoins fort distincts.

Ce guide passe en revue l'état de la connaissance sur les besoins d'éducation des adolescentes des milieux pauvres et met en lumière quelques approches programmatiques parmi les plus prometteuses. II s'inspire largement du compendium d'approches programmatiques récentes énumérées dans New
Lessons: The Power of Educating Adolescent Girls (Lloyd et Young 2009). New Lessons établit les grandes lignes d'un manifeste pédagogique à l'intention des filles, précisant les niveaux de progression et les compétences qu'elles devraient atteindre et acquérir à chaque phase de l'adolescence.

\section{APPROCHES PROMETTEUSES À TESTER ET À DÉVELOPPER}

Il existe de nombreuses et diverses approches politiques et programmatiques conçues pour appuyer l'éducation des adolescentes. Certaines semblent particulièrement créatives et prometteuses. Beaucoup n'ont malheureusement pas été évaluées et ne sont guère connues. Nous nous proposons d'identifier ici quelques-unes des approches les plus prometteuses, ciblées sur les adolescentes déscolarisées en mal d'éducation complémentaire et sur celles inscrites dans l'enseignement formel mais dont il convient de soutenir l'éducation jusqu'au bout de l'adolescence. Le cas échéant, nous recommandons l'essai de ces approches sur base expérimentale avec randomisation, afin d'en démontrer le bien-fondé.

\section{Programmes d'éducation non formelle pour adolescentes déscolarisées \\ Établir et renforcer les filières de scolarisation non formelle à formelle ; mettre l'accent sur les cadettes.}

Les données indiquent clairement l'avantage socioéconomique impressionnant de la scolarisation secondaire formelle des filles (Lloyd et Young 2009). Elles donnent à penser que les programmes non formels devraient s'intéresser davantage à une population plus jeune de filles déscolarisées susceptibles de réintégrer le système formel et d'effectuer la transition vers l'enseignement secondaire formel. Elles préconisent dès lors aussi 


\section{FIGURE 1 TAUX DE SCOLARISATION ET DE PARTICIPATION DES ADOLESCENTS}

À LA VIE ACTIVE, PAR RÉGION ET PAR SEXE

La transition des filles déscolarisées ne se reflète pas de manière équivalente dans celle à la vie active.
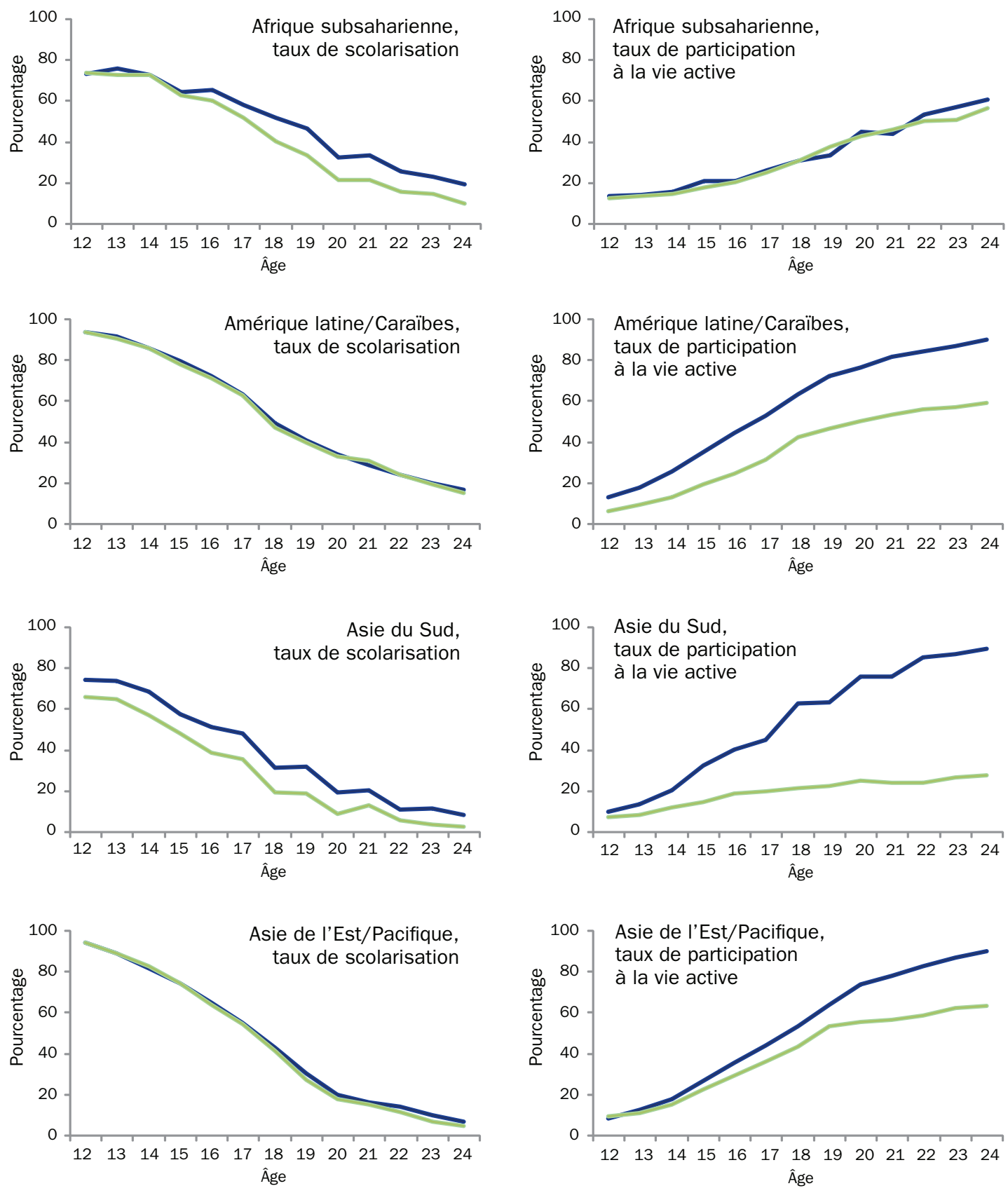

Garçons - Filles

Source: Buvinic, Guzman et Lloyd (2007) 
l'amélioration et l'homologation officielle des programmes d'éducation non formelle, de même que l'établissement de filières de transfert du secteur non formel à l'enseignement formel là où elles n'existent pas encore.

\section{Élargir le soutien de solutions}

d'éducation non formelle à l'objectif de compétences utiles.

De nombreuses ONG et certains gouvernements ont mis au point des programmes d'éducation non formelle à l'intention des adolescentes non scolarisées ou déscolarisées avant d'avoir appris à lire et à écrire ou achevé le niveau d'enseignement primaire. L'objectif est d'apporter une solution d'éducation compensatoire rapide par l'apprentissage de compétences utiles sur le marché, y compris l'alphabétisation. La taille de la population visée augmente avec l'âge des filles et, dans certains contextes parmi les plus pauvres, englobe plus de 50 pour cent des adolescentes. La Figure 2 illustre la taille et la composition (en fonction du niveau d'études atteint) de ce groupe de filles dans une série de pays pauvres. Par exemple, au Mali et au Bangladesh, la majorité des filles sont déscolarisées dès l'âge de 15 ans. Très peu ont achevé l'école primaire et, pour beaucoup, le niveau d'alphabétisme fonctionnel n'est pas atteint (Lloyd et Young 2009).

Étendre l'admissibilité aux subventions conditionnelles et aux bourses (sur la base du besoin et du mérite) aux filles inscrites aux programmes non formels réputés.

Si certains gouvernements l'ont intégrée au sein de leur système au sens large, l'éducation non formelle demeure le plus souvent essentiellement confinée aux ONG dans un contexte extérieur au système ordinaire de l'état. Les filles laissées à la traîne ont généralement le plus besoin d'une assistance financière pour franchir le pas de retour dans le système formel. À ce jour, la plupart des programmes de subventions conditionnelles et de bourses à l'intention des filles restent ciblés sur les filles inscrites dans l'enseignement formel. Là où les solutions d'éducation non formelle sont bien développées, des bourses pourraient être mises à la disposition des filles, sous condition d'acceptation et de fréquentation satisfaisante de l'enseignement formel. On donnerait ainsi aux élèves les plus nécessiteuses et les plus prometteuses l'occasion de réintégrer le système formel au niveau primaire ou secondaire, suivant leurs connaissances et leurs compétences.

De tels programmes feraient du reste sortir de l'ombre l'éducation

\section{MANIFESTE POUR L'ÉDUCATION DES ADOLESCENTES CONNAISSANCES ET COMPÉTENCES QUE LES FILLES DOIVENT ACQUÉRIR À CHAQUE STADE DE L'ADOLESCENCE}

\begin{tabular}{|c|c|c|}
\hline & OÙ CHAQUE FILLE DOIT ÊTRE & CE QUE CHAQUE FILLE DOIT ACQUÉRIR \\
\hline $\begin{array}{l}\text { Début de } \\
\text { l'adolescence } \\
10 \text { à } 12 \text { ans }\end{array}$ & $\begin{array}{l}\text { Enseignement primaire formel ou } \\
\text { enseignement complémentaire } \\
\text { accéléré }\end{array}$ & $\begin{array}{l}\text { Lecture/écriture, calcul, réflexion critique } \\
\text { connaissances d'hygiène élémentaires, } \\
\text { connaissance de la communauté et du } \\
\text { monde }\end{array}$ \\
\hline $\begin{array}{l}\text { Pleine adolescence } \\
13 \text { à } 15 \text { ans }\end{array}$ & $\begin{array}{l}\text { Enseignement post-primaire } \\
\text { formel ou enseignement } \\
\text { complémentaire accéléré }\end{array}$ & $\begin{array}{l}\text { Lecture et écriture courantes pour } \\
\text { un apprentissage durable, réflexion } \\
\text { critique, connaissance courante d'une } \\
\text { langue parlée à l'échelle internationale, } \\
\text { compétences informatiques, compétence } \\
\text { en mathématiques/sciences, } \\
\text { connaissances d'hygiène/santé et } \\
\text { de santé génésique, compétences } \\
\text { financières, compétences propices } \\
\text { à la participation sociale et civique, } \\
\text { connaissance des systèmes sociaux et } \\
\text { des problèmes locaux et mondiaux }\end{array}$ \\
\hline $\begin{array}{l}\text { Fin d'adolescence } \\
16 \text { à } 19 \text { ans }\end{array}$ & $\begin{array}{l}\text { Enseignement secondaire formel } \\
\text { ou éducation non formelle à } \\
\text { vocation professionnelle et de } \\
\text { subsistance }\end{array}$ & $\begin{array}{l}\text { Compétences utiles sur le marché, } \\
\text { capacités et habitudes de collecte } \\
\text { d'information pour un apprentissage } \\
\text { durable, connaissances et compétences } \\
\text { financières }\end{array}$ \\
\hline
\end{tabular}

Source: Lloyd and Young (2009) 


\section{FIGURE 2 POURCENTAGE DE FILLES DÉSCOLARISÉES, EN FONCTION DE L'ÂGE,}

SUIVANT LE PLUS HAUT NIVEAU D'ÉTUDES ATTEINT

Les antécédents d'éducation des adolescentes déscolarisées varient considérablement suivant le pays.
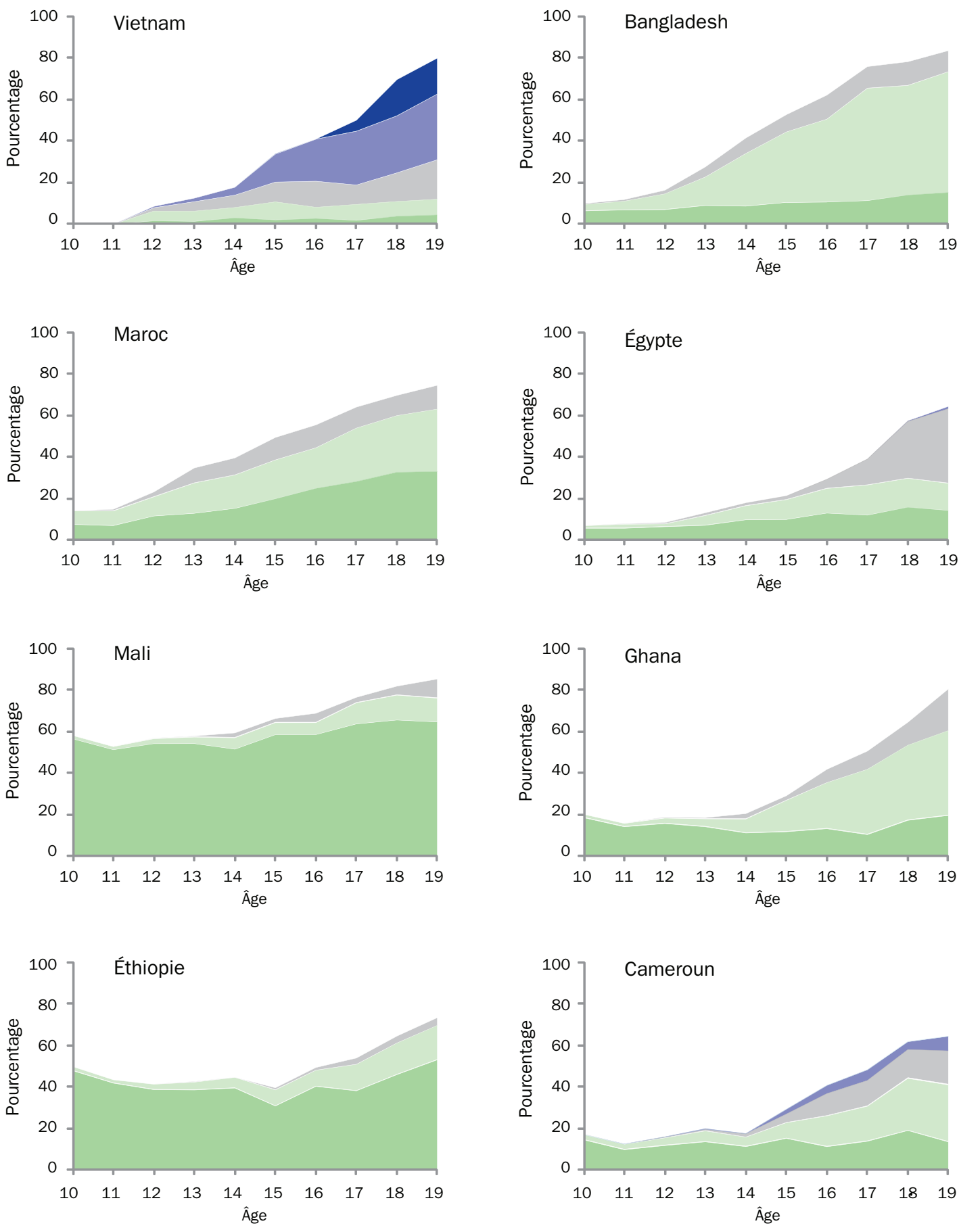

Aucune scolarisation

1 à 4

7 à 9

10 et plus 
non formelle, dont la qualité pourrait être mieux règlementée. Les résultats à évaluer seraient ici les bilans d'apprentissage et d'emploi. L'expérience de BRAC au Bangladesh présente un excellent modèle de bonne intégration des systèmes d'éducation non formelle et formelle.

\section{Collecter les données d'impact} des programmes d'éducation non formelle et les évaluer.

Aucune donnée n'est actuellement disponible sur la participation des adolescentes aux programmes d'éducation non formelle, dont l'impact n'a d'ailleurs pas encore été évalué de manière utile. Plusieurs experts semblent penser que l'inscription à ces programmes est devenue considérable ces dernières années (Lloyd et Young 2009). Dans la plupart des pays, le ministère de l'Éducation ne gère pas ces programmes. La collecte de données d'inscription et d'impact ne relève dès lors pas des systèmes nationaux de gestion de l'information. Les systèmes de collecte secondaires tels que les enquêtes auprès d'échantillons nationalement représentatifs (EDS et MICS, notamment) n'ont du reste pas encore adapté leurs questions relatives à la scolarisation de manière à identifier utilement la participation au système d'éducation non formel.

\section{Politiques et programmes d'éducation utiles aux adolescentes scolarisées}

Encourager l'alignement de l'âge sur le niveau scolaire approprié.

Les Objectifs du Millénaire pour le développement relatifs à l'éducation (OMD 2 et 3) visent l'achèvement de l'enseignement primaire pour tous les enfants et l'élimination de l'écart entre les sexes à tous les niveaux d'éducation. Les progrès réalisés à cet égard depuis 2000 sont considérables. La grande majorité des enfants du monde (filles et garçons) vont aujourd'hui à l'école. Il conviendrait désormais que la communauté internationale embrasse l'objectif plus utile et progressif de la scolarisation universelle à l'âge correspondant au niveau. Le retard de niveau peut résulter du retard de la scolarisation et contribuer à la déscolarisation des filles avant la fin de l'enseignement secondaire. La réalisation de cet objectif plus ambitieux mais critique assurerait que les filles accèdent à l'enseignement secondaire avant la puberté.

À un niveau plus pratique, il est logique de prêter davantage attention aux différences de résultats entre les adolescentes scolarisées avec leur contemporains et celles qui le sont avec d'autres élèves d'âges divers. II n'est en effet pas rare de voir des élèves de 13 ans dans la même classe que d'autres de 18 ans, en Afrique subsaharienne surtout (Lloyd 2010). II semblerait, d'après les données d'Afrique du Sud, que les adolescentes scolarisées au niveau approprié pour leur âge se trouvent souvent confrontées aux avances sexuelles des garçons plus âgés de leur classe, donnant lieu à des bilans de santé génésique (notamment de grossesse) plus défavorables que ceux des filles de la même classe dont l'âge correspond à celui des garçons (Marteleto, Lam, and Ranchhod 2008). La diversité des âges joue aussi un rôle dans les résultats décevants de nombreux programmes de prévention du VIH organisés dans les écoles. II est en effet beaucoup plus difficile d'aborder les sujets sensibles dans les classes dont certains élèves n'ont pas encore atteint l'âge de la puberté alors que d'autres sont déjà mûrs et sexuellement actifs. Ces observations appuient solidement l'idée que les adolescentes pourraient évoluer dans une atmosphère qui leur serait plus favorable dans les contextes scolaires soumis à des normes d'âge plus cohérentes. Dans les grands établissements scolaires (souvent urbains) disposant d'un nombre d'élèves suffisant pour permettre le suivi par âge au niveau de chaque année, un programme pilote randomisé pourrait comparer les bilans de santé et d'apprentissage des adolescentes instruites dans des classes où tous les élèves sont à peu près du même âge par rapport à celles où ils ne le sont pas.

Rendre les écoles primaires et secondaires formelles plus favorables aux filles.

De nombreuses approches propices à l'établissement d'un environnement favorable aux filles existent déjà : certaines ont déjà fait leurs preuves, d'autres semblent prometteuses mais n'ont pas été évaluées et d'autres encore semblent vouées à l'échec. La recherche donne à penser que :

- le recrutement et la formation d'un personnel enseignant de sexe féminin peut être favorable aux filles. Aucune évaluation programmatique n'a cependant envisagé l'efficacité de différentes approches à l'effet dans les contextes où ce personnel demeure minoritaire ;

- le mentoring, le tutorat et le soutien des pairs, de même que la formation antisexiste des enseignants semblent prometteurs, mais de rigoureuses évaluations de ces approches doivent être réalisées ;

- l'offre de toilettes réservées aux filles et d'articles d'hygiène n'améliore vraisemblablement pas l'inscription et la présence des adolescentes à l'école, même si l'on sait que les filles les apprécient. Une analyse des études de recherche récentes ne révèle aucun effet de ces interventions sur les taux d'inscription ou d'abandon de l'école (Lloyd et Young 2009).

Créer des programmes d'activités périscolaires réservés aux filles.

Même quand les adolescentes sont inscrites dans l'enseignement formel (dernier cycle primaire ou niveau secondaire), une plus grande participation aux tâches ménagères est généralement attendue d'elles 
après l'école que de la part de leurs frères scolarisés (Lloyd, Grant et Ritchie, 2008). Cette observation laisse entendre que les adolescentes bénéficieraient plus encore que les garçons de l'occasion de passer un peu de temps personnel en compagnie d'autres filles après les heures d'école ordinaires. Les programmes d'activités périscolaires pour filles pourraient être axés sur les compétences de vie, notamment sur le plan financier et du leadership. Par la même occasion, les aînées pourraient être encouragées à devenir mentors de leurs cadettes. Dans ce contexte, la diversité des âges pourrait être avantageuse, pourvu qu'adéquatement dirigée.

\section{Accroître la " pertinence " féminine de} l'école.

Les questions de pertinence pédagogique transcendent le genre. Compte tenu toutefois du désavantage actuel des jeunes femmes sur le marché de l'emploi, les réformes de l'éducation qui touchent aux questions de pertinence profiteront sans doute différemment aux filles. Par exemple :

- Connaissance de l'anglais et compétences informatiques : les glissements mondiaux de la demande sur le marché de l'emploi ouvrent de nouveaux créneaux aux femmes dans les secteurs des services et du tourisme, notamment, où la connaissance de l'anglais et les compétences informatiques sont souvent recherchées. Si les filles y sont bien préparées à l'école, elles pourront saisir les occasions offertes.

- Compétence financière : les programmes de compétences qui donnent aux filles une idée plus réaliste de leur avenir, concernant notamment la possibilité d'assumer la responsabilité financière de la gestion d'un ménage, présentent une forte motivation d'acquisition de compétences financières élémentaires.
Établir un niveau d'enseignants d'élite, formés et rémunérés davantage, ayant fait preuve de compétences dans les matières "pertinentes".

Les programmes d'enseignement secondaire et la formation des enseignants de ce niveau ne sont pas suffisamment sensibles aux réalités du monde actuel. Même si un nombre grandissant de filles accèdent à l'enseignement secondaire, relativement peu en sont diplômées, en Afrique subsaharienne surtout. Un corps enseignant hautement qualifié constituerait la première étape de la mise en œuvre d'un " programme plus pertinent ". Des incitations spéciales pourraient être offertes aux jeunes femmes pour les encourager à devenir enseignantes d'élite. Les résultats à évaluer couvriraient les compétences d'apprentissage spécifiques visées et le bilan sur le marché de l'emploi pour les enseignants comme pour les élèves.

\section{RESSOURCES : PUBLICATIONS ET PROGRAMMES DIGNES D'INTÉRÊTT}

\section{Publications}

Buvinic, M., J.C., Guzman et C.B. Lloyd. 2007. "Gender shapes adolescence " Development Outreach, June : 12-15. World Bank Institute.

Lloyd, Cynthia B. 2011. " Translating education gains into labor force gains for young women: alternative education strategies ". Document d'information destiné au Rapport Mondial de Suivi sur L'EPT. 2011. Paris : UNESCO.

Lloyd, Cynthia B. 2010. "The role of schools in supporting and promoting sexual and reproductive health among adolescents in developing countries ", dans S. Malarcher (ed.), Social Determinants of Sexual and Reproductive Health: Informing Programmes and Future Research. Genève : Organisation mondiale de la santé, pp. 113-131.

Lloyd, Cynthia B. avec Juliet Young. 2009. New Lessons: The Power of Educating
Adolescent Girls-A Girls Count Report on Adolescent Girls. New York: Population Council.

Lloyd, Cynthia B., Monica J. Grant et Amanda Ritchie. 2008. "Gender differences in time use among adolescents in developing countries: Implications of rising school enrollment rates ", Journal of Research on Adolescence 18(1): 99-120.

Marteleto, Leticia, David Lam et Vimal Ranchhod. 2008."Sexual behavior, pregnancy and schooling among young people in urban South Africa ", Studies in Family Planning 39(4): 351-368.

\section{Programmes non formels prometteurs}

\section{BRAC au Bangladesh et en Afrique}

BRAC est un organisme pionnier d'envergure mondiale, aujourd'hui à la tête du plus vaste système d'enseignement séculaire privé au monde. Le programme BRAC d'éducation primaire non formelle est un modèle grand impact/faible coût d'enseignement d'enfants jamais encore scolarisés ou déscolarisés dès le niveau primaire. À ce jour, près de cinq millions d'enfants, pour la plupart de sexe féminin, ont achevé le programme scolaire BRAC et ont en grande majorité intégré le système d'enseignement public avec, en moyenne, plus de succès que leur pairs ordinairement scolarisés. L'action pédagogique de BRAC a atteint 10 millions d'enfants dans le monde. Pour plus de détails, voir le site Web de BRAC : http://brac.net/ content/what-we-do-education [en anglais].

\section{Ishraq en Égypte}

En Haute Égypte rurale, le projet Ishraq du Population Council intègre les adolescentes déscolarisées dans des espaces d'apprentissage sûrs. Le programme vise l'amélioration des compétences fonctionnelles, financières et de vie des participantes, auxquelles l'occasion sinon rare de faire du sport est également donnée. Les composants du projet se combinent pour apporter aux filles de 
nouvelles compétences valorisées et accroître leur estime personnelle et leur confiance en soi. Pour plus de détails, s'adresser à la haute responsable de programme du Population Council Nadia Zibani (nzibani@popcouncil.org) ou voir le site Web du projet sur http://www.popcouncil.org/projects/ 40_IshraqSafeSpacesGirls.asp.

Youth Education Pack (YEP) du Norwegian Refugee Council dans les régions d'Afrique touchées par un conflit

YEP offre une occasion d'éducation aux jeunes touchés par un conflit et qui, sous l'effet de leur déplacement et de la perte de débouchés, se retrouvent déscolarisés et sans développement de compétences. YEP est un programme d'éducation et de subsistance non formel organisé à temps plein sur une année et axé sur trois composants de poids égal : lecture/écriture/calcul, compétences de vie et compétences professionnelles élémentaires. La priorité est donnée aux jeunes mères célibataires, aux jeunes chefs de ménage et aux jeunes issus des contextes d'éducation les plus démunis. Pour plus de détails, voir le site Web du Norwegian Refugee Council (NRC) : http://www.nrc.no/ ?did=9534911 [en anglais].

\section{Programmes prometteurs au} soutien d'une scolarisation formelle pertinente et favorable aux filles

Developments in Literacy (Pakistan)

Developments in Literacy (DIL) instruit et autonomise les élèves pauvres, en particulier les filles, dans des écoles agencées selon un modèle axé sur l'élève. L'organisation propose aussi un perfectionnement professionnel de qualité aux enseignants et aux directeurs d'école du Pakistan. Pour plus de détails, voir le site Web de l'organisation : http://www.dil.org [en anglais].

\section{Camfed (Campaign for Female Education) en Afrique}

Camfed concentre son effort sur les régions rurales d'Afrique en proie à la pauvreté et à l'exclusion des filles et des jeunes femmes de l'éducation et de ses débouchés. Camfed œuvre au soutien des filles, pour qu'elles puissent fréquenter l'école primaire et secondaire, s'y épanouir et devenir de jeunes adultes aux débouchés prometteurs de formation professionnelle, d'éducation supérieure et de création d'emploi. Pour plus de détails, voir le site Web de l'organisation : http://us.camfed.org [en anglais].

Programmes de scolarisation des filles Room to Read en Asie et en Afrique

Room to Read concentre son action sur l'alphabétisation et l'égalité des sexes dans l'enseignement. L'organisation œuvre en collaboration avec les communautés et les gouvernements locaux d'Asie et d'Afrique pour renforcer les capacités de lecture et d'écriture et former une habitude de lecture au niveau de l'enseignement primaire, ainsi que pour aider les filles à arriver en fin de cycle secondaire dotées de compétences utiles à leur réussite scolaire et au-delà. Pour plus de détails, voir le site Web de l'organisation : http://www.roomtoread.org/page. aspx?pid=284 [en anglais avec accès à une page d'information limitée en français].

\section{Forum for African Women}

Educationalists - Forum des éducatrices africaines (FAWE), écoles sensibles au genre et centres d'excellence en Afrique

FAWE a mis au point le modèle du Centre d'Excellence (COE) à travers lequel les établissements scolaires ordinaires se transforment en écoles sensibles au genre offrant un enseignement de qualité dans le respect des dimensions physiques, pédagogiques et sociales de l'éducation des filles comme des garçons. Pour plus de détails, voir le site Web de l'organisation : http:// www.fawe.org/activities/interventions/ COEs/index.php [en anglais].

Japan Relief for Cambodia/American Assistance for Cambodia (JRfC/AAfC) Girls be Ambitious (Cambodge)

L'objectif du programme Girls be Ambitious - Jeunes filles, ayez de l'ambition est la prévention du trafic des filles et des femmes du Cambodge à des fins d'exploitation sexuelle ou économique, à travers un programme d'incitation encourageant la scolarisation continue des filles des familles pauvres et l'apport à ces filles d'une formation professionnelle supplémentaire qui leur ouvre d'autres débouchés d'emploi, de génération de revenu et d'autonomisation sociale et politique. Pour plus de détails, voir le site Web de l'organisation : http://www. camnet.com.kh/Girls-Ambitious. 


\section{(1) Population Council}

Le Population Council s'attelle aux questions cruciales de la santé et du développement - qu'il s'agisse d'endiguer la progression du VIH, d'améliorer la santé reproductive ou d'offrir aux jeunes la promesse d'une vie satisfaisante et productive. Par ses travaux de recherche en biomédecine, en sciences sociales et en santé publique dans 50 pays, il s'efforce d'apporter avec ses partenaires des solutions qui conduisent à des politiques, des programmes et des technologies plus efficaces, pour améliorer la vie partout dans le monde. Fondé en 1952, le Population Council est une organisation non gouvernementale à but non lucratif. II siège à New York sous la conduite d'un conseil d'administration international. 